\title{
CONJUNCIONES Y LOCUCIONES CONJUNTIVAS CAUSALES EN LAS MEMORIAS DE SEBASTIAN SCHERTLIN VON BURTENBACH.
}

\author{
Julio Sánchez Hernández
}

Sebastian Schertlin von Burtenbach (Schorndorf, 12 Febrero 1496 - Augsburg, 18 Noviembre 1577) vivió una vida de intensa actividad guerrera que le llevaría de simple soldado a una posición sólida e influyente. Su vida militar comenzó a los 23 años y participó en todas las campañas importantes de la época.

Schertlin comenzó a escribir sus memorias a mediados del siglo XVI, usando probablemente diarios de su juventud para el período anterior. En ellas narra no sólo sus campañas militares y las controversias y disputas con sus vecinos sino también los acontecimientos más importantes de su tiempo y de su vida familiar.

De las diferentes ediciones de estas memorias he utilizado en mi trabajo la publicada por Ottmar F. H. Schönhuth ${ }^{1}$. Como dice en su introducción ${ }^{2}$ "Es ist ein urkundlich treuer Abdruck der Handschrift mit ihren Mängeln und Fehlern".

El texto analizado es, por tanto, una autobiografía, escrita durante el siglo XVI y en el ámbito del dialecto suavo.

Sus memorias están escritas en el estadio del idioma alemán conocido como "alto alemán temprano moderno" (Frühneuhochdeutsch).

Se podría pensar que la época del primer alto alemán moderno (1350-1650) -un período con una importancia tan extraordinaria para toda la época moderna- ha sido larga y profundamente trabajada en todos sus aspectos por los estudiosos.

Ciertamente ha sido objeto de numerosos estudios en los últimos 20 años ${ }^{3}$-después de permanecer casi relegado durante muchas décadas-, algunos extraordinariamente

\footnotetext{
${ }^{1}$ Ottmar F. H. Schönhuth, Leben und Thaten des weiland wohledlen und gestrengen Herrn Sebastian Schertlin von Burtenbach, durch ihn selbst deutsch beschrieben. Nach der eigenen Handschrift des Ritters urkundlichtreu. Münster: Druck und Verlang der Aschendirffschen Buchhandlung, 1858.

${ }^{2}$ Schönhuth, op. cit., p. VI.

${ }^{3}$ Destacando entre otros los de:

Hans Eggers, "Zweites Kolloquium `Frühneuhochdeutsch`in Bonn”. En: Deutsche Sprache, 6, 1978, pp. 79-82.
} 
significativos, que hayan ayudado a aclarar el origen de este período de la lengua alemana. Así ha ocurrido en algunos aspectos de la fonología, grafemática y la morfología; mucho menos en la sintaxis y en el léxico ${ }^{4}$.

Mención especial merece la Gramática del Frühneuhochdeutsch de Virgil Moser continuada, pero aún no concluida por Hugo Moser y Hugo Stopp 5 .

Con el estudio de las conjunciones y de las locuciones conjuntivas causales de las memorias de Sebastian Schertlin nos proponemos, en primer lugar, dar la relación de todas las conjunciones y locuciones conjuntivas causales dentro de su contexto y, en segundo lugar, comparar los valores, usos y forma de esas conjunciones y locuciones con otros estudios sobre este tema.

\section{LAS CONJUNCIONES Y LOCUCIONES CONJUNTIVAS CAUSALES.}

als

"doctor Achilles Gasser, [...] sein leibartzet hernach langsam, als er auch kranck gewest, kumen".

Es recogida por Behaghel con valor causal $^{7}$, Rieck la anota también con este valor pero como algo excepcional ${ }^{8}$.

Helmut Graser, "Kolloquium 'Frühneuhochdeutsch'in Bonn" (Februar 1974). En Deutsche Sprache, 1974, pp. 353-358.

Helmut Graser; Klaus Peter Wegera, "Zur Erforschung der frühneuhochdeutschen Flexionomorphologie". En: Zschr. f. Dt. Philologie, 97, 1978, pp. 74-91.

Helmut Henne, "Frühneuhochdeutsch als Aufgabe. Zu einem Kolloquium in Bonn", 21-22, 2, 1974. En: Zschr. f. Germanistische Linguistik, 2, 1974, pp. 87-95.

Oskar Reichmann, "Zweites Bonner Expertenkolloquium 'Frühneuhochdeutsch"”. En: Zschr. f. Germanistische Linguistik, 6, 1978, pp. 63-68.

${ }^{4}$ Ilpo Tapani Piirainen, Frühneuhochdeutsche Bibliographie. Tübingen: 1980.

${ }^{5}$ Virgil Moser, Frühneuhochdeutsche Gramatik t. 1, parte 1. Heidelberg: 1929. T. 1, parte 3. Heidelberg: 1951. (Germanische Bibliothek. I. Reiche: Grammatiken 17; Sprachwissenschaftliche studienbücher).

Hugo Moser; Hugo Stopp, Grammatik des Frühneuhochdeutschen. 1. T., parte 1-3. Heidelberg: 1970-1978. (Germanische Bibliothek. Erste Reihe. Sprachwissenschaftliche Lehr- und Elementarbücher).

${ }^{6}$ Schönhuth, op. cit., p. 71 .

${ }^{7}$ Otto Behaghel, Deutsche syntax. Eine geschichtliche Darstellung, tomo III, Die Satzgebilde. Heidelberg: Carl Winter's Universitätsbuchhandlung, 1928, p. 271.

8 "In wenigen fällen nimmt als kausale Bedeutung an", Susanne Rieck, Untersuchungen zu Bestand und Varianz del Konjunktionen im Frühneuhochdeutsch unter Berücksichtigung der Systementwicklung zur heutigen Norm. Heidelberg: Carl Winter Universitätsverlag, 1977. (Studien zum Frühneuhochdutsch; 2), p. 54. 


\section{allein darum, das}

"dagegen wir vns jrer dapffer erwertt, die jren geschlagen vnd gefangen, allain darumb, das sie vns vnd vnsere vnderthanen vndersteheen mutwilligklich zu verderben""

\section{aus ursach, das}

"Auch sol der her von Liancourt ko. bottschafft meinem konig schriben, [...] vss vrsach, das er wider jr erbeinigung gehandelt"10.

Behaghel recopila la locución aus der ursach, daß como causal ${ }^{11}$.

\section{dann}

"[...] hab ichs annemen wöllen, sann es mir zu bejagen zu weit vnd vngelegen [war]"12.

Esta conjunción está registrada por Zdenek ${ }^{13}$ Behaghel $^{14}$ y Rieck ${ }^{15}$ con valor causal. No la recogen con este valor ni Philipp ${ }^{16}$ ni Otte ${ }^{17}$.

También aparece bajo la forma dan, ambas con gran profusión.

"Insonders so hat mir der ratt vff ain anddres press vom konig an die statt augspurg erkant, [...] dweil ich ain naturalisierter franzos, dan sein Maj. hat mir zuuor ain naturalitet brieff vss gnaden vergunt"18.

\footnotetext{
${ }^{9}$ Schönhuth, op. cit., p. 160.

${ }^{10}$ Schönhuth, op. cit., p. 77.

${ }^{11}$ Behaghel, op. cit., t. III, p. 324.

${ }^{12}$ Schönhuth, op. cit., p. 143.
}

13 Zdenek Masarik, "Zu Bestand und Entwiclungstendenzen der hypotaktischen Konjuntionen des Frühneuhochdeutschen in mähren", pp. 15-57. En: Brünner Beiträge zu Germanistik und Nordistik, tomo IV, Universita J. E. Purkyne V Brne, p. 51.

${ }^{14}$ Behaghel, op. cit., t. III, p. 121.

${ }^{45}$ Con un valor esencialmente comparativo, p. 110. También como causal "denn nur in wenigen unsicheren Ansätzen belegt", Rieck, op. cit., p. 120.

${ }^{16}$ Gerhard Philipp, Einführung ins Frühneuhochdeutsche. Uni-Taschenbücher 822. Heidelberg: Quelle \& Meyer, 1980.

${ }^{17}$ Ruth Otte, Die einleitenden Konjunktionen der Adverbialnebensätze in Sebastian Brants Narrenschiff. Ein Beitrag zur Grammatik des Frühneuhochdeutschen. Inaugural-Dissertation zur Erlangung der Doktorwürde der philosophischen Fakultät der Albert-Ludwings-Universität zu Freiburg im Breisgau. Freiburg/Brsg: 1961.

${ }^{18}$ Schönhuth, op. cit., p. 80 
das

"In der Vasten ist der bischoff von Wirtzburg erschossen worden [...] vnd als man vermaint, das er ainem vom adel genant Wilhelm von Grumbach sein schlösser, [...] eingenomen vnd weder mit noch one recht wider geben wöllen"19.

Behaghel recoge la variante gráfica daz como conjunción introductora de diferentes oraciones subordinadas ${ }^{20}$ entre las que aparecen en último lugar las causales, valor que también anota Rieck ${ }^{21}$.

doch nicht anders, dann

"Solches hab ich [...] angezeigt, doch nicht anders, dann mich langte glauplich ane, das er graf Jgel also verächtlich von jr Maj. reden solte"22.

in dem als

"Vnnd in dem als dem churfürsten zu Saxen gegen hertzog Moritz also, wie oben gemelt, glücklichen ergangen, hat sich der kaiser mit allem hör, [...] zu Vlme erhept"23.

Behaghel registra indem como causal ${ }^{24}$.

sodann

"Vnnd sodann der cardinal von Augspurg mir [...] 24000 fl. [...] schuldig war [...] hab ich

[...] schloss vnnd marckt Buchloe eingenomen"25.

Behaghel $^{26}, \mathrm{Otte}^{27}$ y Rieck ${ }^{28}$ recogen so con valor causal pero no sodann.

sover

"vnd hat jr Maj. mit disen worten geratten [...]; darauf graf gesagt, souer jr Maj. haben wolle ers gern thun"29.

\footnotetext{
${ }^{19}$ Schönhuth, op. cit., p. 114.

20 “Absichtsätzen”, T. III, p. 145, “Bedingungssätzen”, t. III, p. 128, “Behauptungssätzen”, t. III, p. 793, "begründenden Sätzen”, Behaghel, op. cit., t. III, p. 137.

${ }^{21}$ Rieck, op. cit., pp. 97, 98.

${ }^{22}$ Schönhuth, op. cit., p. 138.

${ }^{23}$ Schönhuth, op. cit., p. 64.

24 “Der satz mit indem enthält eine Begründung des Hauptsatzes", Behaghe, op. cit., t. III, p. 191.

${ }^{25}$ Schönhuth, op. cit., p. 37.

${ }^{26}$ Behaghel, op. cit., t. III, p. 288.

${ }^{27}$ Otte, op. cit.; p. 99.

${ }^{28}$ Rieck, op. cit., p. 162.
} 
Behaghel, que registra esta conjunción como sofern, no le atribuye en ningún caso valor causal $^{30}$.

über das

"In disem jar hand mir die von Stain zu Jetingen von ainer holtzfart wegen Jn Schönengeren, über das jch mit jnen am ka. Camergericht rechtshendig bin, abermals bei ka. Maj. vil vngnad u. s. w. angetragen"31.

Behaghel la anota pero no con valor causal ${ }^{32}$.

ursach, das

"her Moritz die statt [...] belegert, aber nit gwinnen mögen, ursach, das Conrat von Hanstein mit 14 fendlein knechten darjnnen war" ${ }^{\text {,33. }}$.

Es recogida por Behaghel con valor causal ${ }^{34}$.

\section{von wegen das}

"[...] hab ich [...] 1200 personen landsknecht [...] zu Burtenpach vnnderhalten, von wegen das allerlei practica von bapst, herzog Heinrichen zu Braunschweik, vnd dem pfaffen schwarm vorhanden waren wider die Euangelischen" ${ }^{35}$.

También aparecen ejemplos en los que la locución está dividida en dos partes, por una coma, de la siguiente forma:

von wegen, das.

"vnnd auch vil stend der Teustschen bewilligen wöllen, von wegen, das es von dess pabsts parthei gar vbersezt vnd vom kaiser zuuoran verordnet war" ${ }^{36}$.

\section{umb das}

"Ist geschenhen umb das sein bruder Ludwing mein mitgsell vnnd hauptman zu Rom gewest als es war wrobert worden"37.

\footnotetext{
${ }^{29}$ Schönhuth, op. cit., p. 142.

30 “sofern erscheint seit den 15. Jh. als Einleitung hypothetischer Sätze", Behaghel, op. cit., t. III, p. 293.

${ }^{31}$ Schönhuth, op. cit., p. 165.

${ }^{32}$ Behaghel, op. cit., t. III, p. 137.

${ }^{33}$ Schönhuth, op. cit., p. 86.

34 "Ursprünglich kan aus der Ursache daß einen begründenden Nebensatz einführen", Behaghel, op. cit., t. III, p. 324.

${ }^{35}$ Schönhuth, op. cit., p. 30.

${ }^{36}$ Schönhuth, op. cit., p. 100.

${ }^{37}$ Schönhuth, op. cit., p. 38.
} 
Behaghel le atribuye valor causal ${ }^{38}$.

\section{umb desswillen das}

"So hat gebracht der churfürst zu Saxen mit yme 13 fendlin [...] knecht [...] vnnd vmb dess willen, das hertzog Henrich zu Braunzweig genante baide Steet geplagt mit allerlai gezäncks dess glaubens halb" ${ }^{\text {"39. }}$.

La partícula causal más común en todas las memorias es weil. Aquí tenemos un período muy interesante porque una sola conjunción introduce siete frases subordinadas causales.

"auch weil die fürsten also spottlich von dem oberland entwichen, die Oberpfaltz vnnd die Thonaw gar vnnd gantz verloren, Wirtenberg vnd obuermelte stett alle bis an Constantz vnd lindaw schantlich vbergeben, Baiernn vnnd Tirol eider vnns, der bischoff von Augspurg vnd Vlme voller feind, darzu mich kainer ainigen menschlichen hilff zu getrosten hette, habe ich mich darein ergebenn" $"$.

Esta conjunción es recogida con esta grafía por Behagel ${ }^{41}$, y Philipp $^{42}$.

Muy frecuente es la variante diweil:

"In selbem zug habend mich die von Nürnberg [...] bestelt, [...] diweil ich aber noch alzu iung vnd vnversucht, haben sie mich meines zusagens auf meine bitt erlassen"43.

Esta forma está a caballo entre weil y las locuciones conjuntivas di wila so, di wila do y die wile daz anotadas por Behaghe ${ }^{44}$ como origen de weil. Philipp ${ }^{45}$ recoge (all(e))dieweil en las que die weil, o sus variantes, entra como primer elemento. También Otte ${ }^{46}$ da esta locución bajo la variante gráfica di wile.

A lo largo de tosa la autobiografía se encuentran muchas subordinadas causales introducidas por otra variante: $d$ weil con la primera $i$ sincopada.

\footnotetext{
${ }^{38}$ Behaghel, op. cit., t. III, p. 137.

${ }^{39}$ Schönhuth, op. cit., pp. 21, 22.

${ }^{40}$ Schönhuth, op. cit., p. 63.

${ }^{41}$ Behaghel, op. cit., t. III, p. 339.

${ }^{42}$ Señala como conjunciones causales weil, sei(n)d/sintemal(en). De estas dos últimas partículas no encontramos en nuestro texto ni in solo ejemplo, así como tampoco en Zdenek y en Otte, Philipp, op. cit., p. 142.

${ }^{43}$ Schönhuth, op. cit., p. 5.

${ }^{44}$ Behaghel, op. cit., t. III, p. 339.

${ }^{45}$ Philipp, op. cit., p. 142.

${ }^{46}$ Otte, op. cit., p. 120.
} 
"Vnnd dweil sich erstlich diser handel liess ansenhenn, [...] wolt mich mein gnediger her zu Hessen nit hinab lassen ziehen" $"$.

Rieck registra die weil -con muchas variantes grafemáticas- como temporal pero no como causal ${ }^{48}$.

Tenemos también frase unidas por coma donde debería ir una conjunción causal:

"aber die vermelte Franzosen sich also hart verschossen, kain pulfer mer gehapt, auch nit vil prouiant, habend nach 7 wochen, als wir die statt belegert, die statt der ka. May aufgeben"49.

\section{CONCLUSIONES.}

En las memorias de Sebastian Scherlin encontramos gran cantidad de conjunciones y locuciones conjuntivas causales; quince en total: allein darum, das; aus ursach, das; dann/dan; das; doch nicht anders, dann; in dem als; sodann; sover; über das; ursach, das; von wegen das / von wegen, das; umb das; umb desswillen das; weil / diweil / dweil.

Hay seis conjunciones: als; dann/dan,; das; sodann; sover; weil / diweil / dweil; una de ellas tiene dos variantes: dann/dan y otra tres: weil /diweil / dweil. Y nueve locuniones conjuntivas: allein darum, das; aus ursach, das; doch nicht anders; dann; in dem als; über das; ursach, das; von wegen das/ von wegen, das; umb das; umb desswillen das.

Seis están recogidas con valor causal en los estudios consultados; cuatro conjunciones: als; dann/dan; das; weil/diweil/dweil y dos locuciones conjuntivas: ursach, das; umb das. $\mathrm{Y}$, de entre ellas, sólo tres conjunciones reunen abundantes ejemplod con este valor en el texto y en la bibliografía ocnsultada: dann/dan; das y weil/dweil/dweil. Las tres restantes: als; ursach, das; umb das; tienen valor causal pero su uso es más restringido.

Otras dos locuciones: in dem als y aus ursach, das están muy próximas a las formas indem y aus der Usache da $\beta$ contrastadas como causales.

Mención aparte merece sondann que parece la unión de dos conjunciones causales: so y dann.

Una conjunción y una locución conjuntiva: sover y über das respectivamente han sido registradas en los estudios consultados pero no como causales sino con otros valores.

Las cuatro locuciones restantes: allein darum, das; doch nicht anders, dann; von wegen das/von wegen, das; umb desswillen das no están registradas en las monografías o artículos

\footnotetext{
${ }^{47}$ Schönhuth, op. cit., p. 32.

48 "Kausale funktion-wie nhd. weil- ist nicht belegt. Doch dürfen wir angesichts des geringen belegmaterials daraus keine generellen Schlüsse für die Sprachepoche ziehen”, Rieck, op. cit., pp. 124-125.

${ }^{49}$ Schönhuth, op. cit., p. 27.
} 
vistos. De éstas, tres tiene en la locución la conjunción das y una dann que funcionan como conjunciones causales independientes.

Estas seis últimas conjunciones y locuciones son, por tanto, el grupo más original e interesante. Están relacionados con los usos más innovadores y personales de Schertlin, con las formas menos comunes.

La autobiografía de Sebastian Schertlin nos da, desde el punto de vista histórico y cultural, una serie de noticias de una época especialmente activa y trascendente y, desde el punto de vista lingüístico, un léxico nuevo, una morfología compleja y una síntaxis rica, variada, original y en buena medida por descubrir.

\section{BIBLIOGRAFÍA}

Behaghel, Otto. Deutsche syntax. Eine geschichtliche Darstellung, tomo III, Die Satzgebilde. Heidelberg: Carl Winter's Universitätsbuchhandlung, 1928.

Hartweg, Frédéric. Wegera, Klaus-Peter. Frühneuhochdeutsch. Eine Einführung in die deutsch Sprache des Spätmittelalters und der frühen Neuzeit. Tübingen: Max Niemeyer Verlag, 1989. (Germanistische Arbeitshefte 33).

Marsarik, Zdenek. "Zu Bestand und Entwiclungstendenzen der hypotaktischen Konjunktionen des Frühneuhochdeutschen in mähren", pp 15-57. En: Brünner Beiträge zu Germanistik und Nordistik, tomo IV, Universita J. E. Purkyne V Brne.

Otte, Ruth. Die einlertenden Konjuntionen der Adverbialnebensätze in Sebastian Brants Narrenschiff. Ein Beitrg zur Grammatik des Frühneuhochdeutschen. InauguralDissertation zur Erlangung der Doktorwüde der philosophishen Fakultät der AlbertLudwigs-Universität zu Freiburg im Breisgau. Freiburg/Brsg: 1961.

Paul, Hermann. Deutsche Grammatik. Tomo IV, parte IV: Syntax. Tübingen: Max Nemeyer Verlag 1986. Unveränderter nachdruck der 1. Auflage von 1920.

Penzl. Herbert. Frühneuhochdeutsch. Bern: Peter Lang, 1984. (Germanistische Lehrbuchsammlung. Herausgegeben von Hans-Gert Roloff. Band 9)

Philipp, Gerhard. Einführung ins Frühneuhochdeutsche Frühneuhochdeutsch. UniTaschenbücher 822. Heidelberg: Quelle \& Meyer, 1980.

Rieck, Susanne. Untersuchungen zu Bestand und Varianz der Konjuntiones im Frühneuhochdeutsch unter Berücksichtigung der Systementwicklung zur heutigen Norm. Heidelberg: Carl Winter Universitätsverlag, 1977. (Studien zum Frühneuhochdeutsch; 2).

Schönhuth, H. Leben und Thaten des weiland wohlendlen und gestrengen Herr Sebastian Schertlin von Burtenbach, durch ihn selbst deutsch beschrieben. Nach der eigenen Handschrift des Ritters urkundlichtreu. Münster: Druck und Verlag der Aschendorffschen Buchhandlung, 1858. 